\title{
Pigmented Villonodular Synovitis Masquerading as Metastasis on PET Imaging
}

\author{
Sadia Choudhery ${ }^{\mathrm{a}}$, Laurice Fischer ${ }^{\mathrm{a}}$, Hythem Omar ${ }^{\mathrm{a}, \mathrm{b}}$
}

\begin{abstract}
Pigmented villonodular synovitis (PVNS) is a metaplastic process that can occur in the synovium, bursae or tendon sheaths and can be localized or diffuse. Its features on radiographs and computed tomography are nonspecific, but magnetic resonance imaging is diagnostic. Its appearance on fluorodeoxyglucose (FDG) positron emission tomography (PET) is not well described. We present a case of recurrent squamous cell carcinoma of the floor of the mouth with an FDG-avid lesion of the left upper arm on a whole-body PET scan that was shown to be PVNS on subsequent biopsy. To our knowledge, this is one of the very few cases reported in the literature describing FDG avidity of PVNS. We explore the features of PVNS on various imaging modalities and its potential for being mistaken for malignancy.
\end{abstract}

Keywords: PVNS; Cancer; PET; MRI

\section{Introduction}

Pigmented villonodular synovitis (PVNS), benign hypertrophy of the synovium, was originally described in 1852 [1]. Since then, various terms have been used to describe this entity. In 1941, Jaffe and colleagues proposed the term PVNS [2]. PVNS can be focal or diffuse and can present as intra-articular or extra-articular forms. The knee joint is most commonly affected by the diffuse intra-articular form of PVNS whereas the hand joints are most commonly affected by the extra-articular form [3]. PVNS appears to be a metaplastic

\footnotetext{
Manuscript accepted for publication February 11, 2014

${ }^{\mathrm{a} D e p a r t m e n t ~ o f ~ R a d i o l o g y, ~ U n i v e r s i t y ~ o f ~ T e x a s ~ S o u t h w e s t e r n ~ M e d i c a l ~}$ Center, Dallas, TX, USA

${ }^{\mathrm{b}}$ Corresponding author: Hythem Omar, Department of Pathology, University of Texas Southwestern Medical Center, 5323 Harry Hines Blvd., Dallas, TX 75390, USA.

Email: hythem.omar@utsouthwestern.edu
}

doi: http://dx.doi.org/10.14740/jmc1691w process, but its etiology remains undetermined.

Radiography and computed tomography (CT) show nonspecific features including joint effusions, focal soft tissue masses or bone erosions. On magnetic resonance imaging (MRI), PVNS is typically intermediate to dark on T1and $\mathrm{T} 2$-weighted images and blooms due to the presence of hemosiderin on gradient imaging. Since fluorodeoxyglucose (FDG) positron emission tomography (PET) is not used for the diagnosis of PVNS, its appearance on PET is not well described. We present a case of recurrent squamous cell carcinoma of the floor of the mouth with an FDG-avid lesion of the left upper arm on a whole-body PET scan that was shown to be PVNS on subsequent biopsy.

\section{Case Report}

A 53-year-old male with a prior history of left floor of the

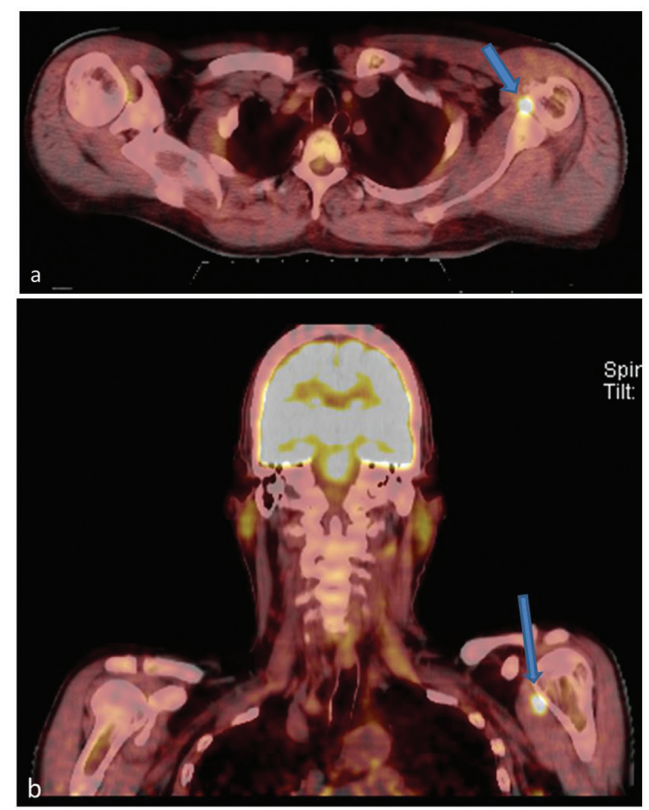

Figure 1. ${ }^{18} \mathrm{~F}-\mathrm{FDG}$ PET axial (a) and coronal (b) images show a focal region of increased uptake (arrows) just medial to the left humeral head. The SUV maximum of this lesion was 11.6. 


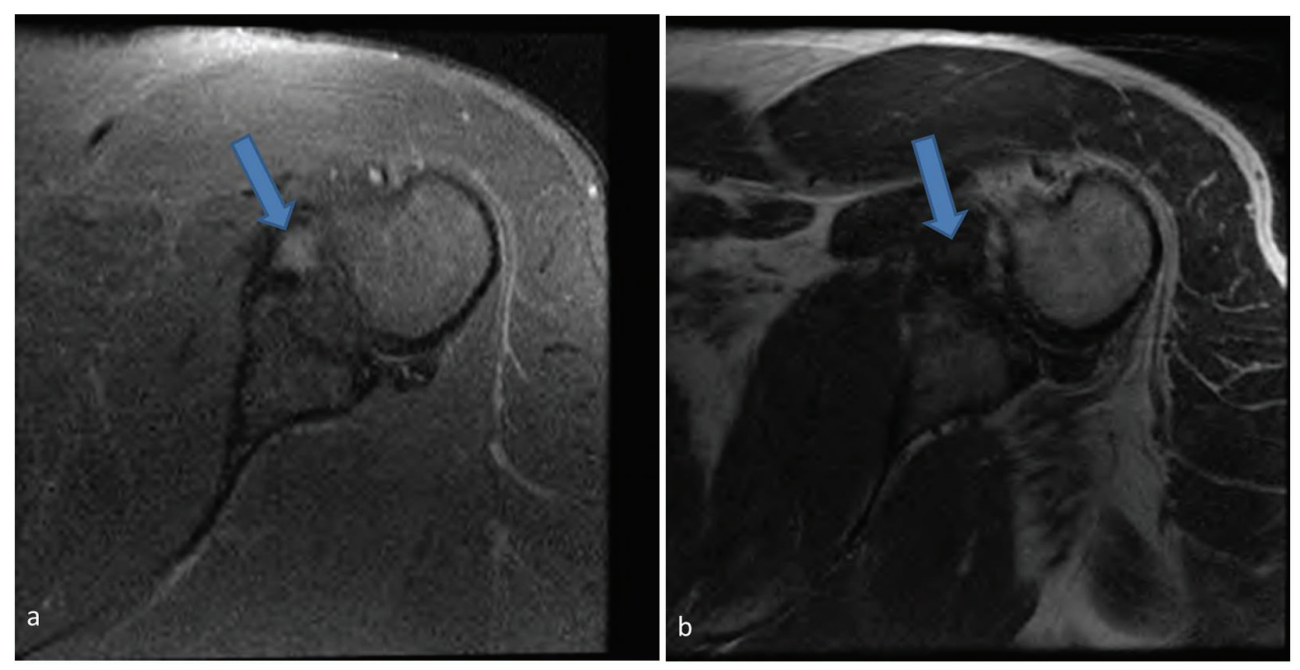

Figure 2. Axial STIR (a) and T1 (b) magnetic resonance images show a focal region of intermediate STIR and low T1 signal (arrows) medial to the humerus corresponding to the focus of increased uptake on PET scanning.

mouth squamous cell carcinoma treated with wide local excision, left neck dissection and a radial forearm free flap presented for a follow-up. A cervical nodal mass was discovered on physical examination and a CT scan of the neck revealed recurrent left base of the tongue mass. A biopsy of this mass was consistent with recurrent squamous cell carcinoma.

A whole-body PET scan ordered to evaluate the extent of disease showed a focus of intense FDG uptake just medial to the left humeral head concerning for metastasis (Fig. 1a, b). Maximum standardized uptake value (SUV) was 11.6. A subsequent MRI demonstrated a $9 \times 5 \mathrm{~mm}$ intra-articular nodular focus abutting the humeral head, deep to the subscapularis tendon. This lesion exhibited intermediate shorttau inversion recovery (STIR) and low T1 signal (Fig. 2a,

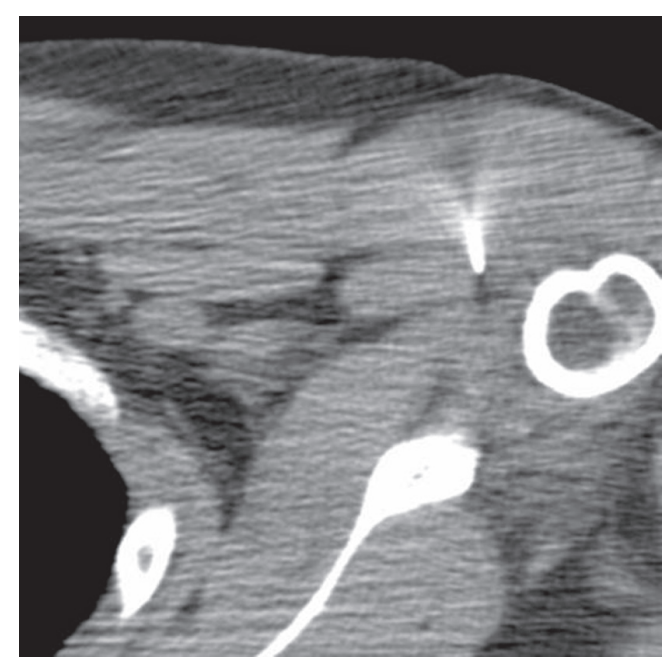

Figure 3. Axial CT image during percutaneous biopsy showing the needle tip directed toward the area of interest. b). The case was discussed in a multidisciplinary conference where it was decided that a biopsy would allow definitive staging of the patient's disease and thereby determine his treatment/prognosis. Pathology from a CT-guided fine needle aspiration and core biopsy (Fig. 3) showed multinucleated giant cells and macrophages amidst a background of abundant synovial cells and hemosiderin, consistent with a diagnosis of PVNS (Fig. 4a, b).

\section{Discussion}

PVNS is categorized by the World Health Organization as localized giant cell tumor of the tendon sheath or diffuse intra-articular involvement. It usually presents in the third to fifth decades of life with various clinical symptoms including pain, soft-tissue mass, swelling and joint dysfunction [3]. The etiology of PVNS is unknown, but it has been attributed to an inflammatory process, repetitive trauma, repeated hemorrhage, or a lipid metabolism disorder [4]. A neoplastic cause has recently been postulated, given its association with cytogenetic abnormalities [5].

PVNS lesions are pale and multicolored with irregular villous or nodular projections [6]. Histologically, proliferative synovial-like mononuclear cells, fibrous stromal cells, foam and hemosiderin-laden cells, and multinucleated giant cells are seen [6]. Radiographs may be normal or may show a soft tissue mass or a joint effusion. With intra-articular involvement, erosions may be seen on both sides of the joint [3]. CT findings are nonspecific, also showing effusions with or without erosive changes. MRI is diagnostic with intermediate-low signal intensity foci with or without joint fluid and "blooming" on gradient-echo images due to the presence of hemosiderin [3]. PVNS is known to be hypervascular and 


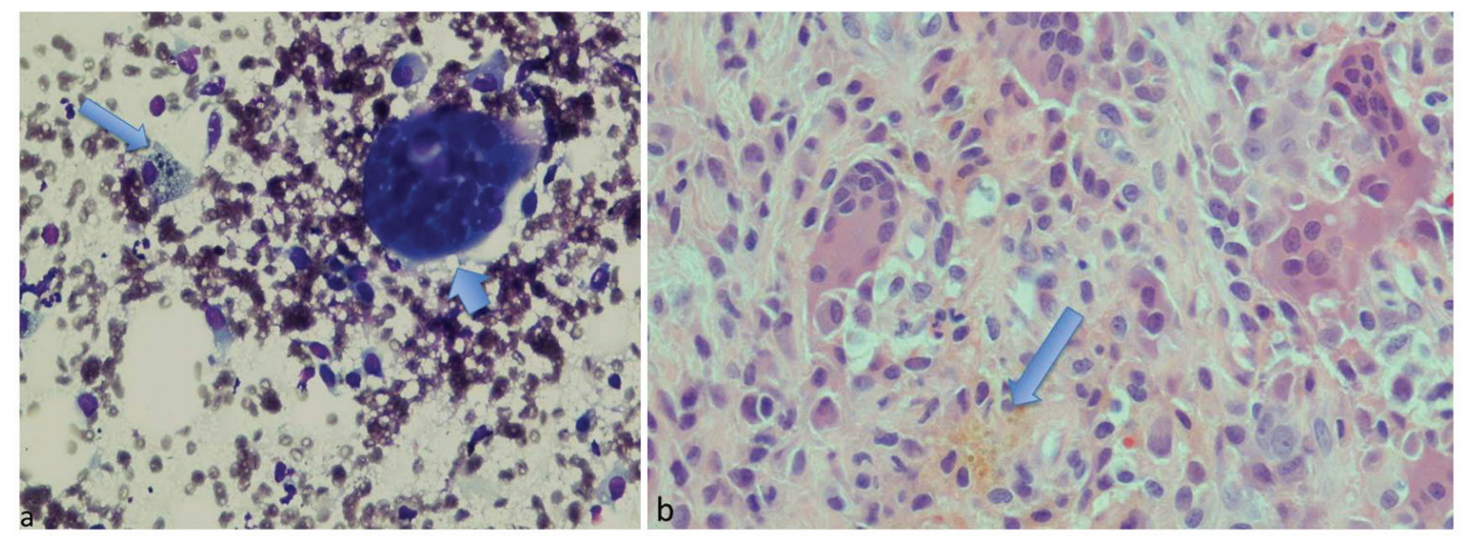

Figure 4. (a) Diff-Quick stained touch preparation shows multinucleated giant cells (short arrow) and macrophages (long arrow) within a background of abundant, small synovial-like cells. (b) The hematoxylin and eosin stained core biopsy tissue contains similar findings, in addition to hemosiderin (arrow).

hypercellular, leading to increased uptake on Tc-99m MDP, Tc-99m DMSA and gallium-67 citrate [7]. The appearance of PVNS on PET scans is not well described as this modality is not routinely used for evaluation. PVNS has been incidentally diagnosed on FDG PET scans done for evaluation of metastatic disease. It has been shown to be hypermetabolic with maximum SUV values of up to 11.3 reported in the literature [8]. Our case had a slightly higher SUV value of 11.6. In some studies, PVNS has also been shown to respond to cancer treatment with subsequent decrease in FDG activity [4]. These findings can complicate evaluation of a patient with a known malignancy, especially in the setting of neoplasms such as melanoma or sarcoma which can develop musculoskeletal metastatic disease [4].

Depending on the form (localized vs. diffuse), radiation may be employed in conjunction with surgical excision. For diffuse pathology, synovectomy is usually necessary. Failure to achieve complete resection can lead to disease recurrence.

A key distinguishing feature of PVNS is its intra-articular location. It is exceedingly rare for neoplasm, primary or metastatic, to be within a joint. Primary lung cancer is the most likely to metastasize to the synovium [9]. Sarcoma or melanoma can also produce intra-articular metastases, but oropharyngeal cancers (as in our case) are not known to do so. Additionally, only a few primary malignancies are intra-articular including synovial cell sarcomas and synovial chondrosarcomas [9].

Other intra-articular lesions such as amyloid would also be a consideration in the setting of renal failure. At times, gouty tophi or rheumatoid nodules may present similarly in the appropriate clinical setting of the arthropathy. Intra-articular hemorrhage from hemophilia can present with similar signal characteristics and blooming on gradient imaging depending on the age of the clot.

With increasing utilization of PET imaging for oncological workup, it is likely that incidental non-neoplastic pathol- ogy will become increasingly evident and may confound patient workup and treatment planning. While hot on PET scanning, clues to the diagnosis of PVNS include intermediate to low magnetic resonance signal characteristics, blooming on gradient imaging and intra-articular location.

\section{References}

1. Chassaignac M. Cancer de la gaine des tendons. Gas Hosp Civ Milit. 1852;47:185-190.

2. Jaffe HL, Lichtenstein L, Sutro CJ. Pigmented villonodular synovitis, bursitis and tenosynovitis. Arch Pathol . 1941;31:731-765.

3. Murphey MD, Rhee JH, Lewis RB, Fanburg-Smith JC, Flemming DJ, Walker EA. Pigmented villonodular synovitis: radiologic-pathologic correlation. Radiographics. 2008;28(5):1493-1518.

4. Amber IB, Clark BJ, Greene GS. Pigmented villonodular synovitis: dedicated PET imaging findings. BMJ Case Rep. 2013;2013.

5. McGrath J, Monu J. Pigmented Villonodular Synovitis Imaging. Medscape.com. Accessed 12/5/13. www.medscape.com.

6. Stevenson JD, Jaiswal A, Gregory JJ, Mangham DC, Cribb G, Cool P. Diffuse pigmented villonodular synovitis (diffuse-type giant cell tumour) of the foot and ankle. Bone Joint J. 2013;95-B(3):384-390.

7. Nguyen BD. PET, CT, and MR imaging of extra-articular pigmented villonodular synovitis. Clin Nucl Med. 2007;32(6):493-495.

8. Kitapci MT, Coleman RE. Incidental detection of pigmented villonodular synovitis on FDG PET. Clin Nucl Med. 2003;28(8):668-669.

9. Sheldon PJ, Forrester DM, Learch TJ. Imaging of intraarticular masses. Radiographics. 2005;25(1):105-119. 